\title{
PRETEXT Stage 3 Hepatoblastoma
}

National Cancer Institute

\section{Source}

National Cancer Institute. PRETEXT Stage 3 Hepatoblastoma. NCI Thesaurus. Code C7141.

An internationally developed presurgical anatomic staging system using imaging techniques: Tumor involves 3 adjoining quadrants or 2 nonadjoining quadrants; 1 quadrant or 2 nonadjoining quadrants are free of tumor. (from PDQ 2004) 